\title{
Lung ultrasound findings in patients with novel SARS-CoV-2
}

\author{
Mark E. Haaksma (ib) ${ }^{1,2,3}$, Micah L.A. Heldeweg ${ }^{1,2,3}$, Jorge E. Lopez Matta ${ }^{3,4}$, \\ Jasper M. Smit ${ }^{1,2,3}$, Jessica D. van Trigt ${ }^{1,2}$, Jip S. Nooitgedacht ${ }^{1,2}$, \\ Carlos V. Elzo Kraemer ${ }^{3,4}$, Mark van de Wiel ${ }^{5}$, Armand R.J. Girbes ${ }^{1,2}$, \\ Leo Heunks ${ }^{1,2}$, David J. van Westerloo ${ }^{3,4}$ and Pieter R. Tuinman ${ }^{1,2,3}$
}

Affiliations: 'Dept of Intensive Care Medicine, Amsterdam University Medical Centers, VUmc, Amsterdam, The Netherlands. ${ }^{2}$ Amsterdam Cardiovascular Sciences Research Institute, Amsterdam University Medical Centers, Amsterdam, The Netherlands. ${ }^{3}$ Amsterdam Leiden Intensive Care Focused Echography, Amsterdam, The Netherlands. "Dept of Intensive Care Medicine, Leiden University Medical Center, Leiden, The Netherlands. ${ }^{5}$ Dept of Epidemiology and Data Science, Amsterdam University Medical Centers, VUmc, Amsterdam, The Netherlands.

Correspondence: Mark E. Haaksma, VU University Medical Center Amsterdam, Postbox 7507, 1007MB, Amsterdam, The Netherlands. E-mail: m.haaksmadamsterdamumc.nl

\section{ABSTRACT}

Background: Over 2 million people worldwide have been infected with severe acute respiratory distress syndrome-coronavirus-2 (SARS CoV-2). Lung ultrasound has been proposed to diagnose and monitor it, despite the fact that little is known about the ultrasound appearance due to the novelty of the illness. The aim of this manuscript is to characterise the lung ultrasonographic appearance of critically ill patients with SARS-CoV-2 pneumonia, with particular emphasis on its relationship with the time course of the illness and clinical parameters.

Methods: Adult patients from the intensive care unit of two academic hospitals who tested positive for SARS$\mathrm{CoV}-2$ were included. Images were analysed using internationally recognised techniques which included assessment of the pleura, number of B-lines, pathology in the PLAPS (posterolateral alveolar and/or pleural syndrome) point, bedside lung ultrasound in emergency profiles, and the lung ultrasound score. The primary outcomes were frequencies, percentages and differences in lung ultrasound findings overall and between short ( $\leqslant 14$ days) and long (>14 days) durations of symptoms and their correlation with clinical parameters.

Results: In this pilot observational study, 61 patients were included with 76 examinations available for analysis. $26 \%$ of patients had no anterior lung abnormalities, while the most prevalent pathological ultrasound findings were thickening of the pleura $(42 \%), \geqslant 3$ B-lines per view $(38 \%)$ and presence of PLAPS (74\%). Patients with "long" duration of symptoms presented more frequently with a thickened and irregular pleura (32 (21\%) versus 11 (9\%)), C-profile (18 (47\%) versus $8(25 \%))$ and pleural effusion (14 (19\%) versus $3(5 \%)$ ), compared to patients with short duration of symptoms. Lung ultrasound findings did not correlate with arterial oxygen tension/inspiratory oxygen fraction ratio, fluid balance or dynamic compliance.

Conclusion: SARS-CoV-2 results in significant, but not specific, ultrasound changes, with decreased lung sliding, thickening of the pleura and a B-profile being the most commonly observed. With time, a thickened and irregular pleura, C-profile and pleural effusion become more common findings. When screening patients, a comprehensive ultrasound protocol might be necessary.

@ERSpublications

Lung ultrasound is a valuable tool for detection and monitoring of pulmonary changes in \#SARSCoV2 infection https://bit.ly/3lfWrUo

Cite this article as: Haaksma ME, Heldeweg MLA, Lopez Matta JE, et al. Lung ultrasound findings in patients with novel SARS-CoV-2. ERJ Open Res 2020; 6: 00238-2020 [https://doi.org/10.1183/ 23120541.00238-2020].

This trial was registered in the Netherlands Trial Register (www.trialregister.nl/) with registration identifier NL8540. Data will be shared upon reasonable request with other researchers in the field.

Received: 2 May 2020 | Accepted after revision: 25 Sept 2020

Copyright $\odot$ ERS 2020. This article is open access and distributed under the terms of the Creative Commons Attribution Non-Commercial Licence 4.0. 


\section{Introduction}

At the time of writing, close to 2.5 million people worldwide have been infected with severe acute respiratory distress syndrome-coronavirus-2 (SARS-CoV-2), of whom approximately 150000 have died. The rapid spread of the illness in the pandemic necessitates constant adjustments of clinical and management protocols in keeping with our evolving knowledge of the illness. As part of this, there has been ongoing debate as to the optimal approach for imaging these patients as the normal gold standard for thoracic imaging, chest computed tomography (CT), poses the risk of spread of infection since it necessitates transportation of patients. Furthermore, given the novelty of the illness, the sensitivity and specificity of CT when diagnosing SARS-CoV-2 is untested.

Lung ultrasound has significant advantages as the imaging modality of choice in both diagnosis and monitoring; it has high sensitivity for detecting pathology at the lung surface, such as pleural thickening, consolidation and ground-glass like patterns as seen on CT [1,2]. Recent literature also demonstrates that ultrasound outperforms chest radiography in detecting these pathologic entities [3]. Furthermore, lung ultrasound has additional advantages in that it offers no radiation exposure, does not require transport and, therefore, also saves valuably needed personal protective equipment.

However, due to the novelty of the disease, there is a scarcity of data related to the typical lung ultrasound findings which may be observed in patients infected with SARS-CoV-2. In addition, we do not know if lung ultrasound can be used for monitoring of disease progression, as it is unknown how findings may change throughout the course of the disease and if they correlate to clinically relevant disease-related parameters.

Therefore, we aim to present an outline of lung ultrasound findings in critically ill SARS-CoV-2 patients overall, in relation to duration of symptoms, to determine if there is a correlation between ultrasound findings and physiological parameters, such as the arterial oxygen tension/inspiratory oxygen fraction $(\mathrm{P} / \mathrm{F})$ ratio.

\section{Methods}

\section{Study design and population}

This study was conducted in two academic intensive care units (ICUs) at Amsterdam University Medical Centers (UMC) (Vrije Universiteit Medical Center (VUmc), Amsterdam, the Netherlands) and Leiden University Medical Center (LUMC; Leiden, the Netherlands). The protocol to utilise data gathered during routine ultrasound was approved by the local ethics board (registration ID 2020.011). The necessity for informed consent was waived. The trial was registered with the Netherlands Trial Register (registration ID NL8540). Patients were followed up until discharge, death or up until submission of the article, if the patients were still admitted on the ICU.

The study population consisted of adult (aged >18 years) ICU patients, who tested positive for SARS-CoV-2 at least once before admission. In addition to baseline demographics, days from hospital admission and ICU admission, time spent on the ventilator before ultrasound examination, Sequential Organ Failure Assessment score on the day of examination, ventilator settings, inflammatory markers, and serum creatinine were recorded. Data were derived form a dedicated patient data management system and data closest to the time of examination were used.

Two groups were defined based on symptom duration from their onset, where $\leqslant 14$ days was defined as the "short group" and $>14$ days as the "long group", which were arbitrarily chosen based on the clinical observation that the disease often worsens after 10-14 days.

\section{Ultrasound measurements}

All images were acquired by lung ultrasound certified clinicians, using a Sonosite-EDGE II or Philips Lumify ultrasound system. Certification involved a 2-day course and thereafter supervision by a physician with extensive ultrasound experience ( $>5$ years) until sufficient expertise was reached (a minimum of 30 exams) [4]. Researchers (M.E. Haaksma, M.L.A. Heldeweg and J.E. Lopez Matta) performed offline ultrasound analysis and were blinded to the patient's baseline characteristics.

The examinations in both centres were performed with the patient in the supine position, utilising the BLUE (bedside lung ultrasound in emergency) protocol: two ventral-lateral (upper BLUE point and lower BLUE point) and one dorso-lateral point (PLAPS (posterolateral alveolar and/or pleural syndrome) point of measurement(s), on either side of the thorax) [5].

Ventral measurements were performed using a 10-5 MHz linear transducer (VUmc) or a 12-4 MHz linear transducer (LUMC), both in lung setting (suppression of artefact filtering software) and with image depth set at $>6 \mathrm{~cm}$. This depth was chosen based on the hospital's local guideline on ultrasound acquisition to 
ensure standardisation of imaging [6]. The PLAPS measurements were made with a 5-1 MHz cardiac transducer (VUmc) or a $4-1 \mathrm{MHz}$ broadband phased array transducer (LUMC), with settings freely adjustable by the operators in both centres to obtain an ideal image. In one centre (LUMC), in addition to the aforementioned protocol, the Lung Ultrasound Score (LUS), a 12-region protocol was performed as well [7]. In this centre the number of B-lines were not routinely measured due to presumed low reproducibility [8].

In each image the following analyses were made: 1) movement of pleura classified as present (easily and directly visible), present but subtle (visible, but closer examination needed) or absent; 2) the pleura was described as either normal, thickened (by evaluating if the pleura appeared as a sharp, thin line or wider with a diffuse border), irregular (by evaluating if it was one continuous line or had small echogenic bands/ septa/gaps in between) or thickened and irregular; 3) the total number of B-lines; 4) the appearance of $\mathrm{B}$-lines as either separated or confluent; 5 ) the appearance of the lung parenchyma per view as normal, $\geqslant 3$ B-lines and consolidated; 6) the BLUE profile as A, A/B, B or C (A' and B' were not present); 7) in case of a C-profile, as small or large consolidation (small in case of shred-sign and large if a tissue like pattern was visible); and 8) PLAPS as either absent or present with consolidation and/or pleural effusion [7, 9]. Due to the hierarchical nature of the BLUE protocol, where a C-profile overrules a B-profile, which

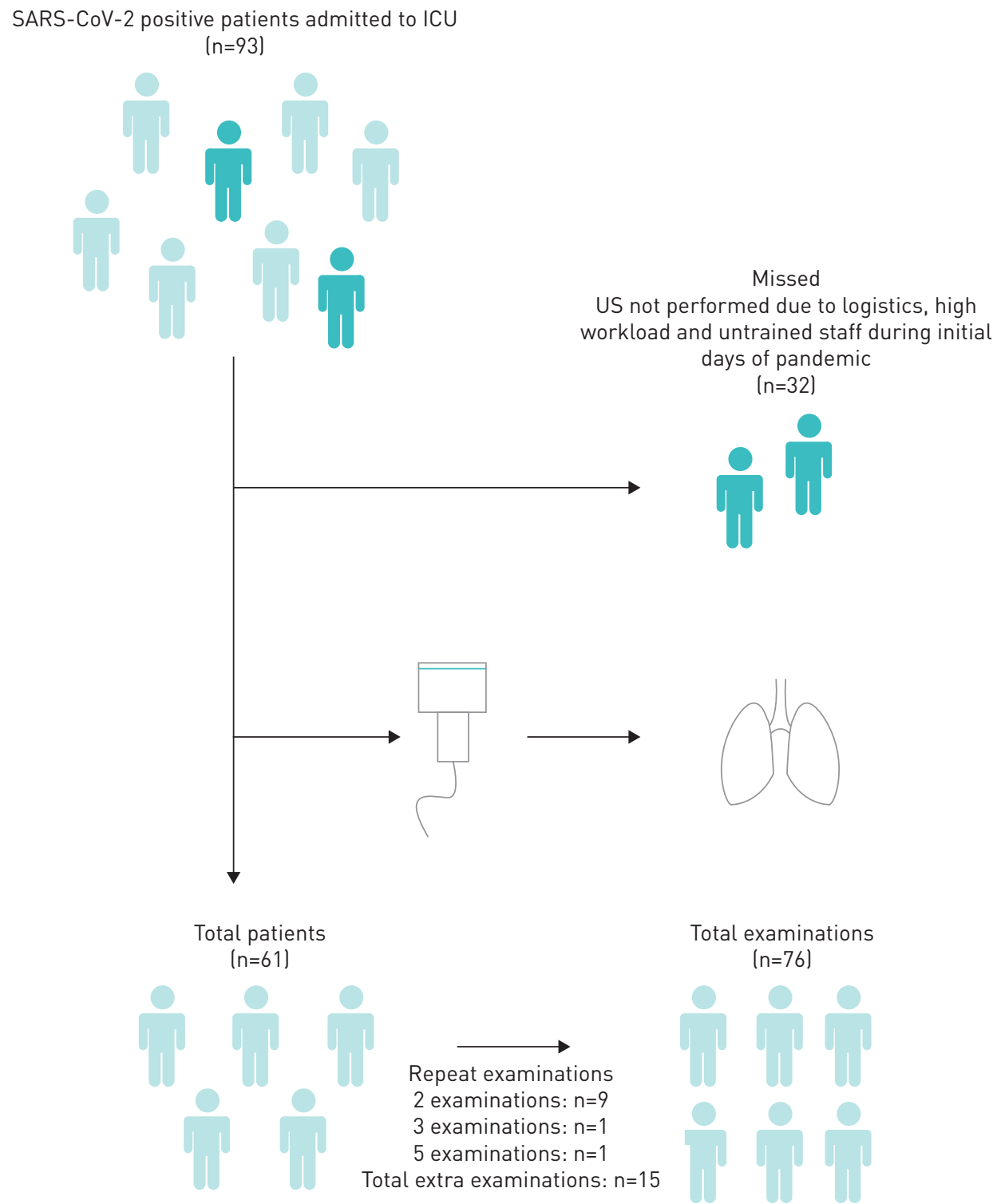

FIGURE 1 Flowchart of patient inclusion. SARS-CoV-2: severe acute respiratory syndrome-coronavirus-2; ICU: intensive care unit; US: ultrasound. 
overrules an A-profile, data were presented per view and per examination, with the aim of better capturing the heterogeneity of the disease.

\section{Statistical analysis}

No sample size calculation was performed as this study was meant to be a pilot exploratory study to give insights into baseline findings in patients with SARS-CoV-2. Statistical analyses were performed using SPSS IBM version 22 and R statistical software. Variables were tested for normality with the Shapiro-Wilk test, evaluation of histograms and Q-Q plots. Descriptive statistics are presented as mean $\pm \mathrm{SD}$, median (interquartile range) or $\mathrm{n}(\%)$ when appropriate. Differences in characteristics between duration of symptoms/ventilation, and the $\leqslant 14$ days and $>14$ days of symptoms groups were tested with an independent samples t-test, Mann-Whitney U-test or Pearson's Chi-squared test, using Yates' continuity correction when appropriate. The latter was used to test a global association between the categories of the variable (e.g. A, B, C) and the binarised duration. To complement this global p-value, we also provide confidence intervals for difference in proportions (long versus short duration), specifically per category. These were based on continuity corrected Z-statistics. Correlations for dichotomous and categorical variables were tested with generalised linear models and linear regression for continuous variables.

Analyses were made per patient and view, equalling six views per patient. The LUS was calculated based on six views per hemithorax but analysed as one data-point per patient.

In one centre (LUMC) the total amount of B-lines per view was not counted and perceived as missing data, while in the other centre (VUmc) the LUS was not calculated and perceived as missing data.

\section{TABLE 1 Baseline characteristics of included patients}

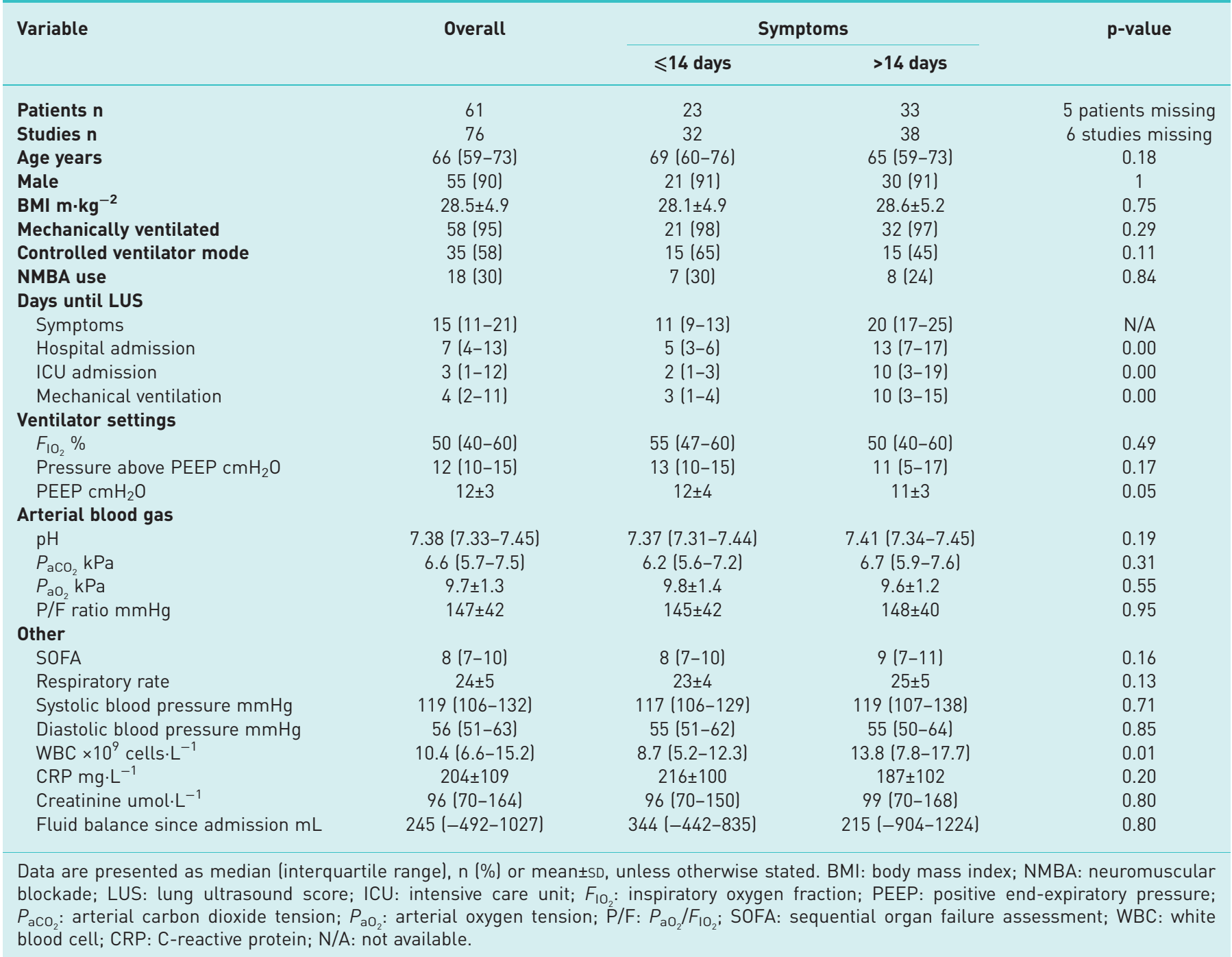


Statistical analyses were performed using two-sided hypothesis tests. A p-value $<0.05$ was regarded as statistically significant.

\section{Results}

This study was performed from 27 March 2020 until 20 April 2020. Patient enrolment is summarised in figure 1. A total of 93 patients were screened of which 61 patients were included, with a total of 76 lung ultrasound examinations $((50 \times 1$ exam $)+(9 \times 2$ exams $)+(1 \times 3$ exams $)+(1 \times 5$ exams $))$ and 456 images/views analysed. Of these, $13(2.9 \%)$ were missing. When divided into short and long duration of symptoms, five patients (30 views $(6.7 \%$ of all views)) were not included due to missing information on the start date of the symptoms.

\section{Baseline characteristics}

Baseline characteristics are presented in table 1 . An overall body mass index (BMI) of $28.5 \pm 4.9 \mathrm{~m} \cdot \mathrm{kg}^{-2}$ was found and $90 \%$ of the included patients were male. No differences were found in baseline characteristics between the missed and included patients (BMI 28.6 $\pm 3.5 \mathrm{~m} \cdot \mathrm{kg}^{-2}(\mathrm{p}=0.15), 81 \%$ male $(\mathrm{p}=0.22)$ and aged $62(54-70)$ years $(\mathrm{p}=0.15))$. Patients in the long duration group had a longer hospital admission time, ICU admission time and mechanical ventilation time before ultrasound examination and a higher white blood cell count $\left(8.7(5.2-12.3)\right.$ versus $13.8(7.8-17.7)$ cells $\left.\cdot \mathrm{L}^{-1}, \mathrm{p}=0.01\right)$.

\section{Ultrasound analysis}

Ultrasound variables per view are presented in table 2 and per patient in table 3. Lung sliding was present in $99 \%$ of patients but was only subtle in $35 \%$ of views and did not differ between groups. An unaffected pleura was seen in $36 \%$ of views, with a thickened pleural line being seen in $42 \%$ of them. A thickened and irregular pleura was more frequently seen in the long versus short symptom duration group, 32 (21\%) versus $11(9 \%)$, respectively ( $\mathrm{p}=0.024)$. According to the BLUE protocol, an A-, A/B-, B- and C-profile was seen in $26 \%, 21 \%, 15 \%$ and $38 \%$ of patients, respectively. The C-profile was seen more frequently in the long symptom duration group and consolidation per view, 8 (25\%) versus 18 (47\%) (difference $-12,95 \%$ CI -48-2) and 11 (9\%) versus 31 (20\%) (difference -11, 95\% CI -21-3), respectively. Overall, the PLAPS

\section{TABLE 2 Lung ultrasound findings in critically ill patients with SARS-CoV-2 pneumonia}

\begin{tabular}{|c|c|c|c|c|c|c|c|}
\hline \multirow[t]{2}{*}{ Variable } & \multirow[t]{2}{*}{ Overall } & \multirow[t]{2}{*}{ Missing } & \multicolumn{2}{|c|}{ Symptoms } & \multirow[t]{2}{*}{ Difference $(95 \% \mathrm{Cl})$} & \multirow[t]{2}{*}{ p-value } & \multirow[t]{2}{*}{ Missing } \\
\hline & & & $\leqslant 14$ days & $>14$ days & & & \\
\hline Lung sliding $\mathbf{n}$ & 303 & 1 & 127 & 152 & & 0.609 & 25 \\
\hline Present but subtle & 105 (35) & & 42 (33) & 59 (39) & $-6(-17-6)$ & & \\
\hline Absent & $3(1)$ & & $1(1)$ & $1(1)$ & $\mathrm{N} / \mathrm{A}$ & & \\
\hline Pleura n & 303 & 1 & 127 & 152 & & 0.024 & 25 \\
\hline Irregular & $16(5)$ & & $6(5)$ & $7(5)$ & $0(-5-5)$ & & \\
\hline Thickened and irregular & $48(16)$ & & $11(9)$ & $32(21)$ & $-12(-21-4)$ & & \\
\hline Lung $\mathbf{n}$ & 303 & 1 & 127 & 152 & & 0.004 & 25 \\
\hline Normal & $139(46)$ & & $71(56)$ & 59 (39) & $17(5-29)$ & & \\
\hline$\geqslant 3$ B-lines & 117 (38) & & 45 (35) & $62(41)$ & $-6(-17-7)$ & & \\
\hline Consolidation & $47(15)$ & & $11(9)$ & $31(20)$ & $-11(-21-3)$ & & \\
\hline Small & $44(92)$ & & $10(91)$ & $21(95)$ & $-4(-28-19)$ & & \\
\hline Large & $4(8)$ & & 1 (9) & $1(5)$ & $\mathrm{N} / \mathrm{A}$ & & \\
\hline PLAPS type $n$ & 139 & 13 & 56 & 73 & & 0.051 & 23 \\
\hline No pathology & 54 (39) & & $25(45)$ & $23(32)$ & $7(5-50)$ & & \\
\hline Pleural effusion & $17(12)$ & & $3(5)$ & $14(19)$ & $-14(-23-20)$ & & \\
\hline Consolidation & $64(46)$ & & $25(45)$ & $35(48)$ & $-3(-22-16)$ & & \\
\hline Pleural effusion and consolidation & 4 (3) & & $3(5)$ & $1(1)$ & $\mathrm{N} / \mathrm{A}$ & & \\
\hline
\end{tabular}

Data are presented as $\mathrm{n}(\%)$, unless otherwise stated. SARS-CoV-2: severe acute respiratory syndrome-coronavirus-2; PLAPS: posterolateral alveolar and/or pleural syndrome; N/A: not available. 
point did not show pathology in $39 \%$ of views. When present, consolidation (46\%) was the most frequent finding. Pleural effusions were seen more frequently in the long symptom group (3 (5\%) versus 17 (19\%); difference $-14,95 \%$ CI $-23-20)$. Out of all examinations, only one examination (1.3\%) did not show any detectable ultrasonographic changes. A "typical" ultrasound exam is presented in figure 2.

The LUS was calculated in 24 patients, with a mean of $19 \pm 1$. The score did not differ between symptom duration groups. There was no correlation between BLUE profile or LUS with $\mathrm{P} / \mathrm{F}$ ratio $(\mathrm{p}=0.29)$, fluid balance $(p=0.84)$ or dynamic compliance $(p=0.19)$. For the LUS, a correlation with compliance $\left(\mathrm{R}^{2}=0.27\right.$, $\mathrm{p}=0.02)$ and trend for fluid balance $(\mathrm{p}=0.09)$ was observed, but there was no correlation with $\mathrm{P} / \mathrm{F}$ ratio $(\mathrm{p}=0.98)$.

\section{Discussion}

The main findings of this exploratory study are as follows. 1) SARS-CoV-2 results in significant changes in the lung in most patients, which are detectable on ultrasound with subtle lung sliding, pleural thickening and a C-profile being the most frequently observed, though with a large proportion of cases still show an A-profile. 2) In patients with long duration of symptoms (>14 days) compared to those with a short duration ( $\leqslant 14$ days), a thickened and irregular pleura, C-profile and pleural effusion are more common. 3) The BLUE profiles and LUS did not show a strong correlation with P/F ratio, fluid balance or dynamic compliance.

In recent weeks, several case-reports have already described changes of the pulmonary parenchyma due to SARS-CoV-2. However, these were only presented in a very small number of patients without baseline characteristics or a standardised ultrasound approach $[2,10]$. To our knowledge, this is the first study to accurately present a comprehensive overview of ultrasound findings in a large cohort.

In line with the published data, we noticed a high prevalence of pleural changes, with more than half of the patients presenting with either thickening and/or irregularities. Additionally, a relatively high frequency of anterior interstitial syndrome and lung consolidation was noted, which was also described in the other studies. However, while pleural effusion was uncommon in the short symptom duration group, it became more prevalent in the long symptom duration group. The latter finding is not entirely in line with the previous literature; however, in those studies no time course was described which makes direct comparison difficult.

In comparison to other pathological entities, these findings resemble those seen in acute respiratory distress syndrome, with varying patterns of interstitial syndrome and consolidation, though with a lower rate of pleural effusion $[11,12]$. In line with this, it should be noted that while the described findings are typically seen in patients with SARS-CoV-2, they are not specific for the condition. This is especially relevant in a first-line setting, where different aetiologies could present with similar findings.

Comparison of our data with regards to the temporal component with other ultrasound studies is not possible, as this data is not yet available. What was observed in our data set was a change in the

\section{TABLE 3 Lung ultrasound findings in critically ill patients with SARS-CoV-2 pneumonia per patient ${ }^{\#}$}

\begin{tabular}{|c|c|c|c|c|c|c|c|}
\hline \multirow[t]{2}{*}{ Variable } & \multirow[t]{2}{*}{ Overall } & \multirow[t]{2}{*}{ Missing } & \multicolumn{2}{|c|}{ Symptoms } & \multirow[t]{2}{*}{ Difference $(95 \% \mathrm{CI})$} & \multirow[t]{2}{*}{ p-value } & \multirow[t]{2}{*}{ Missing } \\
\hline & & & $\leqslant 14$ days & $>14$ days & & & \\
\hline BLUE per case $n$ & 76 & 0 & 32 & 38 & & 0.055 & 6 \\
\hline $\mathrm{A} / \mathrm{B}$ & $16(21)$ & & $7(22)$ & $9(24)$ & $-2(-23-20)$ & & \\
\hline B & $11(15)$ & & $4(13)$ & $6(16)$ & $-3(-22-16)$ & & \\
\hline C & $29(38)$ & & $8(25)$ & $18(47)$ & $-12(-48-2)$ & & \\
\hline PLAPS n & 68 & 8 & 27 & 37 & & 0.661 & 12 \\
\hline Present & $50(74)$ & & $19(70)$ & $29(78)$ & $-8(-33-17)$ & & \\
\hline Absent & $18(26)$ & & $8(30)$ & $8(22)$ & $8(-17-33)$ & & \\
\hline Lung scores calculated $\mathbf{n}$ & 24 & 52 & 10 & 14 & & 0.244 & 52 \\
\hline LUS & $19 \pm 1$ & & $18 \pm 3$ & $20 \pm 6$ & & & \\
\hline
\end{tabular}

Data are presents as $\mathrm{n}(\%)$ or mean \pm SD, unless otherwise stated. SARS-CoV-2: severe acute respiratory syndrome-coronavirus-2; BLUE: bedside lung ultrasound in emergency; PLAPS: posterolateral alveolar and/or pleural syndrome; LUS: lung ultrasound score. ${ }^{\#}$ : data are presented as 76 examinations in 61 patients. 
FIGURE 2 Lung ultrasound findings in patients with severe acute respiratory syndrome-coronavirus-2 pneumonia. Right lung upper image indicates BLUE: B-profile with irregular and thickened pleura. Right lung lower image indicates BLUE: A-profile with 2 B-lines, PLAPS: consolidated lung with pleural effusion. Left lung upper image indicates BLUE: A-profile with some pleural thickening. Left lung lower image indicates BLUE: C-profile larrow: Shred sign), PLAPS: no pathological findings, unaffected lung moving. Stars indicate B-lines and arrows indicate pleural effusion. Lu: lung; Li: liver; D: diaphragm, S: spleen

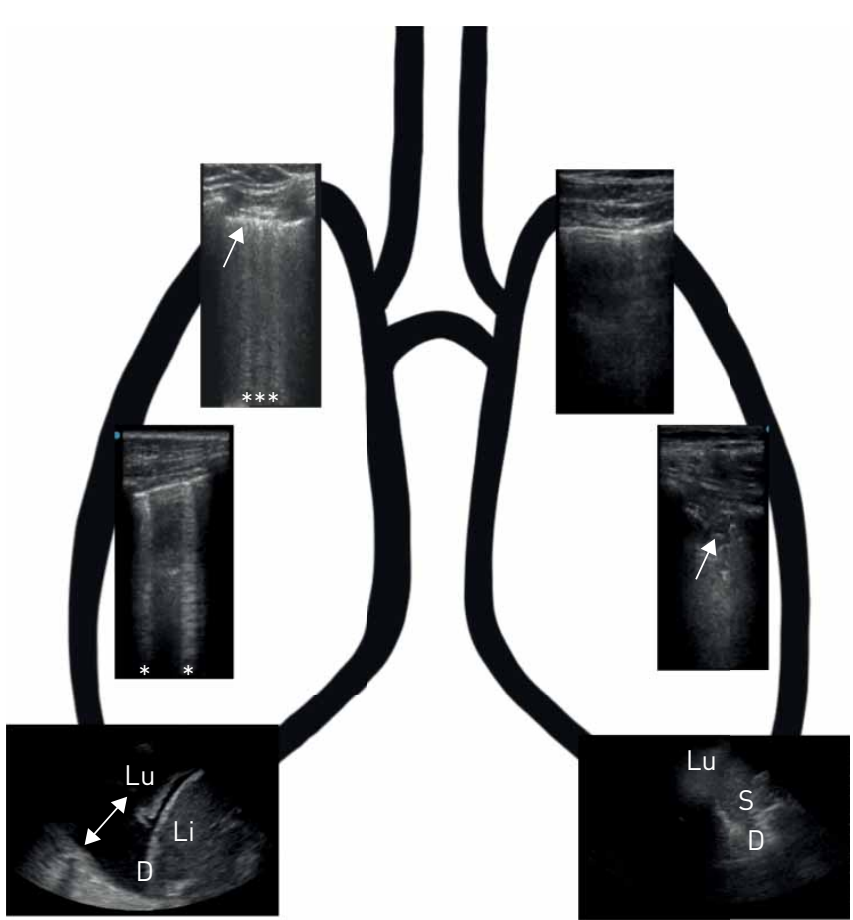

occurrence of the described findings over time, with increasing frequency of pleural thickening and irregularity, C-profile and pleural effusion. Interestingly, these changes overlap with previous articles that demonstrated comparable changes on CT [13-15]. With this in mind, we believe lung ultrasound presents a valuable alternative for monitoring disease progression in the context of this pandemic [16]. Especially considering that it does not require transport and therefore not only saves urgently needed personal protective equipment but also limits the necessity to take patients out of isolation. However, it is crucial to realise that $26 \%$ of all cases were found to have an A-profile, indicating a non-pathological state of the lungs when assessing the anterior BLUE points. This is not completely unexpected, given that on CT the lung parenchyma is very heterogeneously affected and pathological regions might be missed. At this point, we wish to mention that in all but one case with A-profile, either thickening of the pleura, present PLAPS or one BLUE point showing a B-line pattern was seen. It is therefore an important consideration, when utilising lung ultrasound examinations as a diagnostic modality in patients with suspected SARS-CoV-2 infection, to select a more comprehensive approach such as the 12-region protocol or to also interpret pleural thickening or a local B-line pattern as indicative of disease [7]. We hypothesise that this becomes especially relevant in patients presenting to the emergency ward, as in this population abnormalities in the PLAPS point or pleural thickening might be less frequently encountered, thus leaving an important number of patients with a negative lung ultrasound examination.

We also set out to determine whether the BLUE profiles or LUS were correlated to currently relevant parameters such as the P/F ratio, fluid balance or dynamic lung compliance. This was not the case, except for LUS and compliance, where there was a very weak correlation and therefore not regarded as relevant. We hypothesise that this might be attributed to several factors. While the 12-region protocol covers a larger area of the lung than the BLUE protocol, a large part of the most dorsal regions is not visualised and therefore not accounted for. In addition, even if extensive, lung ultrasound only examines the outermost parts of the pulmonary parenchyma, while studies show that on CT deeper lung parts are also affected [13-15]. This issue is also relevant when considering the radiographic differences between the proposed "H-" and "L-type" lungs, as the former can present with deep and subpleural abnormalities, while the latter mostly with subpleural abnormalities [17]. Therefore, one might under- or overestimate disease severity in the $\mathrm{H}$ - and L-type respectively, solely based on ultrasound examination, as it cannot visualise deeper structures. It is important to note that as no sample size calculation was performed, these conclusions should be read with caution and analyses in a larger dataset is necessary.

\section{Strengths}

The strength of this study is its size with an overview of lung ultrasound findings in SARS-CoV-2 positive patients and reporting both on the use of the BLUE protocol and LUS. This contributes greatly to the 
currently available body of evidence. In addition, the study was carried out in two different hospitals, by multiple operators and using two different ultrasound approaches, thereby increasing its external validity. It is also the first study to highlight ultrasonographic differences according to symptom duration.

\section{Limitations}

This study has several important limitations. First, no sample size calculation was performed, for which reason caution is warranted when interpreting results due to significant results by chance. The included sample was based on availability of an ultrasound examination and in the first days of the outbreak with a large number of admissions, restructuring of the wards and medical support by other specialties untrained in lung ultrasound, some patients did not receive an ultrasound examination. With the introduction of better logistics and dedicated proning teams this changed and we were able to examine every admitted patient. Secondly, the length of symptom duration for subgroup analysis was arbitrarily chosen, based on our experience of clinical worsening after 10-14 days. Thirdly, some patients were examined more than once, which could introduce bias. However, the images acquired showed great heterogeneity within patients over time, limiting this effect to some extent. In addition, due to grouping by symptom duration, these cases were distributed between groups, also mitigating this effect. Fourthly, we were not able to correlate our findings to end-points such as mortality or extubation outcome, as the majority of the patients were still admitted and ventilated at the time of writing. Finally, $90 \%$ of the included patients were male, which limits the overall generalisability. Still, this number roughly reflects the percentage of male patients admitted to our ICUs during this pandemic.

\section{Conclusion}

SARS-CoV-2 pneumonia results in significant changes in the lung, detectable on ultrasound with decreased lung sliding, pleural thickening and a B-profile being the most frequently observed. Over time, a thickened and irregular pleura, C-profile and pleural effusion become more prevalent. Lung ultrasound might be a valuable alternative to CT in diagnosing and monitoring SARS-CoV-2 pneumonia. When screening patients, a comprehensive ultrasound protocol should be used.

Acknowledgements: Mark E. Haaksma takes responsibility for the content of the manuscript, including the data and analysis. We would like to extend our gratitude to Adrian Wong (Dept of Intensive Care, King's College Hospital, London, UK) for proofreading our manuscript.

Author contributions: M.E. Haaksma, M.L.A. Heldeweg, A.R.J. Girbes, L. Heunks, C.V. Elzo Kraemer, J.E. Lopez Matta, D.J. van Westerloo and P.R. Tuinman were responsible for the conception and design of the work. M.E. Haaksma, M.L.A. Heldeweg, J.S. Nooitgedacht, J.D. van Trigt, J.E. Lopez Matta and P.R. Tuinman were responsible for acquisition and/or analysis of the data. M.E. Haaksma, M.L.A. Heldeweg and J.E. Lopez Matta were responsible for building the database. M.E. Haaksma and P.R. Tuinman were responsible for drafting the manuscript and all authors provided critical revisions for it. All authors read and approved the final manuscript, and ensured that questions related to the accuracy or integrity of any part of the work were investigated and resolved.

Ethics approval and consent to participate: Approval was given by the local ethics committee (Medisch Ethische Toetsings Commissie, VUmc, Amsterdam, the Netherlands) with the study number 2020.11. Consent for participation was not applicable as ultrasound measurements were carried out as part of routine clinical examination.

Conflict of interest: None declared.

\section{References}

1 Soldati G, Smargiassi A, Inchingolo R, et al. Is there a role for lung ultrasound during the COVID-19 pandemic? J Ultrasound Med 2020; 39: 1459-1462.

2 Peng Q-Y, Wang X-T, Zhang L-N. Findings of lung ultrasonography of novel corona virus pneumonia during the 2019-2020 epidemic. Intensive Care Med 2020; 46: 849-850.

3 Winkler MH, Touw HR, van de Ven PM, et al. Diagnostic accuracy of chest radiograph, and when concomitantly studied lung ultrasound, in critically ill patients with respiratory symptoms: a systematic review and meta-analysis. Crit Care Med 2018; 46: e707-e714.

4 Touw HRW, Tuinman PR. Lung ultrasound: routine practice for the next generation of internists. Neth $J$ Med 2015; 73: 8 .

5 Lichtenstein DA, Mezière GA. Relevance of lung ultrasound in the diagnosis of acute respiratory failure the BLUE protocol. Chest 2008; 134: 117-125.

6 Neuteboom O, Heldeweg MLA, Pisani L. Assessing extravascular lung water in critically ill patients using lung ultrasound - a systematic review on methodological aspects in diagnostic accuracy studies. Ultrasound Med Biol 2020; 46: 1557-1564.

7 Bouhemad B, Mongodi S, Via G, et al. Ultrasound for "lung monitoring" of ventilated patients. Anesthesiology 2015; 122: 437-447.

8 Haaksma ME, Smit JM, Heldeweg MLA, et al. Lung ultrasound and B-lines: B careful!. Intensive Care Med 2020 46: 544-545. 
9 Dietrich CF, Mathis G, Cui X-W, et al. Ultrasound of the pleurae and lungs. Ultrasound Med Biol 2015; 41: 351-365.

10 Buonsenso D, Piano A, Raffaelli F, et al. Point-of-care lung ultrasound findings in novel coronavirus disease-19 pnemoniae: a case report and potential applications during COVID-19 outbreak. Eur Rev Med Pharmacol Sci 2020; 24: 2776-2780.

11 Chiumello D, Marino A, Cressoni M, et al. Pleural effusion in patients with acute lung injury: a CT scan study. Crit Care Med 2013; 41: 935-944.

12 Lichtenstein D, Grenier P. Comparative diagnostic performances of auscultation, chest radiography, and lung ultrasonography in acute respiratory distress syndrome. Anesthesiology 2004; 100: 7.

13 Pan F, Ye T, Sun P, et al. Time course of lung changes on chest CT during recovery from 2019 novel coronavirus (COVID-19) pneumonia. Radiology 2020; 295: 715-721.

14 Bernheim A, Mei X, Huang M, et al. Chest CT findings in coronavirus disease-19 (COVID-19): relationship to duration of infection. Radiology 2020; 295: 200463.

15 Zhou Z, Guo D, Li C, et al. Coronavirus disease 2019: initial chest CT findings. Eur Radiol 2020; 30: 4398-4406.

16 Rubin GD, Ryerson CJ, Haramati LB, et al. The role of chest imaging in patient management during the COVID-19 pandemic: a multinational consensus statement from the Fleischner Society. Chest 2020; 158: 106-116.

17 Gattinoni L, Chiumello D, Caironi P, et al. COVID-19 pneumonia: different respiratory treatments for different phenotypes? Intensive Care Med 2020; 46: 1099-1102. 